\title{
ADULT REFERENCE LEVELS IN DIAGNOSTIC AND INTERVENTIONAL RADIOLOGY FOR TEMPORARY USE IN SWITZERLAND
}

\author{
A. Aroua ${ }^{1, *}$, A. Besançon ${ }^{1}$, I. Buchillier-Decka ${ }^{1}$, P. Trueb ${ }^{2}$, J.-F. Valley ${ }^{1}$, F. R. Verdun ${ }^{1}$ and W. Zeller ${ }^{2}$ \\ ${ }^{1}$ University Institute of Applied Radiation Physics, Grand-Pré 1, 1007 Lausanne, Switzerland \\ ${ }^{2}$ Swiss Federal Office of Public Health, 3003 Bern, Switzerland
}

Received November 23 2003, amended May 3 2004, accepted May 202004

\begin{abstract}
This work aims at establishing a set of diagnostic reference levels (DRLs) for various types of examinations performed in diagnostic and interventional radiology. The average doses for 257 types of radiological examinations were established during the 1998 nationwide survey on the exposure of the Swiss population by radiodiagnostics. They were calculated using appropriate dosimetric models and average technical parameters. The DRLs were derived from the average doses using a multiplying factor of 1.5. The DRLs obtained were rounded and compared to the data reported in the literature. The results are in most cases comparable to the DRLs determined by the 3rd-quartile method. These discrepancies registered in some cases, particularly for complex examinations, can be explained by significant differences in the protocols and/or the technical parameters used. A set of DRLs is proposed for a large number of examinations to be used in Switzerland as temporary values until a national dosimetric database is set up.
\end{abstract}

\section{INTRODUCTION}

The definition, establishment and implementation of diagnostic reference levels (DRL) have become in recent years a central issue in the management of the radiation dose delivered to the patient in diagnostic and interventional radiology. Several scientific meetings showed an increasing interest for this subject such as the Workshops of $1993^{(1)}$, Luxembourg in $1997^{(2)}$, Malmö in $1999^{(3)}$ and Dublin in $1999^{(4)}$, the Hiroshima IRPA-10 Congress in $2000^{(5)}$, the Malaga IAEA Conference in $2001^{(6)}$. The European Radiation Protection, Education and Training Organisation (ERPET) has dedicated one of its training sessions (Passau, 2000) to the establishment of DRLs ${ }^{(7)}$, while the Applied Radiation and Isotopes journal has published a whole issue on patient doses in diagnostic radiology ${ }^{(8)}$.

The historical evolution of the DRL concept and the various quantities proposed are described by different authors ${ }^{(9,10)}$. Several works reported in the literature in recent years focused on the review of the different concepts related to DRLs ${ }^{(11)}$, their role, usefulness, impact and associated problems ${ }^{(12)}$, the various dosimetric quantities considered ${ }^{(13)}$, and the practical difficulties encountered in the establishment and implementation of DRLs ${ }^{(14-22)}$. There is a growing need for harmonisation of the concepts and quantities ${ }^{(23)}$. The different approaches to DRLs at the international level have been summarised by the ICRP in a recent report ${ }^{(24)}$.

*Corresponding author: abbas@aroua.com
Setting up DRLs is relatively easy in the case of simple examinations such as conventional radiographic views. However, for complex examinations such as dose-intensive procedures involving fluoroscopy or CT (computed tomography) examinations, establishing a DRL is a difficult task due to the large variability of the fluoroscopy time and the number of images (number of series and slice number and thickness in case of CT) leading to a wide distribution of patient doses. This is due to several factors including the often loose definition of the examination, differences in the techniques and protocols used, the complexity of the case and the experience of the radiologist.

In general, the DRLs are based on dosimetric surveys. The 3rd-quartile method has been proposed about a decade ago in the UK by the NRPB and is now widely used ${ }^{(25)}$. This method prescribes the use as a DRL of a dose value corresponding to $3 / 4$ of the dose distribution established by the survey. The NRPB issued recently the 2000 review of the DRLs recommended for use in the UK and based on this $\operatorname{method}^{(26)}$.

In the absence of a dose distribution, another method is suggested consisting in multiplying the established average dose by an appropriate factor. The surveyed dose distributions are often extended to the right side. A recent limited investigation which covered a couple of Swiss hospitals and a few examinations indicated that the ratio between $75 \%$ and the mean value of the dose distribution lies in most cases between 1.2 and 1.3 but can reach 1.5 in a few cases. To be on the conservative side the factor to be applied in this work is 1.5 . This method and the 


\section{A. AROUA ET AL}

1.5-factor were used in a recent work to establish DRLs for cardiology at the European level ${ }^{(27)}$.

In Switzerland no recent dose distributions are available. Those proposed by Mini as a basis for the establishment of national DRLs ${ }^{(28)}$ were determined in 1992 and cover a small number of types of examinations only. In addition, more recent dose distributions were established for a few examination types: four angiographic examinations at the Bern University Hospital(29), and ten examinations including fluoroscopy, angiography and coronary dilation at the Lausanne University Hospital(30). Moreover, a methodology was recently proposed for the establishment of DRLs for paediatric $\mathrm{CT}^{(31)}$.

The Swiss strategy for establishing DRLs consists in the following elements:

(1) Launching nationwide dosimetric surveys in order to establish national DRLs for the various radiological modalities. A first dosimetric survey, carried out in 2002, focused on CT examinations (10 hospitals were covered and 15 CT examinations investigated). The second survey, carried out in 2003, focused on doseintensive fluoroscopic examinations (five university hospitals were covered and eight diagnostic and interventional examinations investigated).

(2) Until the results of these surveys are available, European DRL values are adopted when possible. This is the case of a number of radiographic examinations for which the DRL values proposed at the European level are adopted in Switzerland: skull, cervical spine, chest, thoracic spine, abdomen, lumbar spine, pelvis and hip. A directive was issued by the Swiss Federal Office of Public Health concerning these DRLs and containing the following information: definitions, proposed DRLs, application of DRLs, measurement of the entrance surface dose
(ESD), calculation of the ESD. Moreover, a computer software was developed allowing the practitioners to assess online the ESD for a given radiographic examination and to compare it with the corresponding DRL.

(3) In the absence of European data, temporary DRLs are calculated from the average doses established in the framework of the 1998 nationwide survey on the exposure of the public by radiodiagnostics in Switzerland.

This paper presents the work undertaken in order to establish a set of provisional DRLs for various types of examinations performed in diagnostic and interventional radiology in Switzerland, based on average patient effective doses.

\section{METHODS}

Average-dose values are available in Switzerland for 257 types of examinations covering the various modalities of diagnostic and interventional radiology. They have been established by dosimetric modelling considering average technical parameters for each type of examination, in the framework of the 1998 nationwide survey on the exposure of the Swiss population by medical radiology $(32,33)$.

Various operational dose quantities are used to establish DRLs depending on the radiological modality considered as presented in Table 1.

In the case of a radiographic projection, the operational dose quantity is the ESD (mGy), which takes this empirically based form:

$$
\mathrm{ESD}=K \cdot\left(\frac{U}{100}\right)^{2} \cdot \frac{3}{F_{\mathrm{A}}} \cdot Q \cdot \frac{1}{(\mathrm{FSD})^{2}} \cdot \mathrm{BSF},
$$

where $U(\mathrm{kV})$ is the tube voltage, $Q(\mathrm{mAs})$ is the tube current-time product, $F_{\mathrm{A}}$ is the filtration expressed

Table 1. Dosimetric quantities used to establish the diagnostic reference levels.

\begin{tabular}{|c|c|c|c|}
\hline Modality & Dosimetric quantity & Abbreviation & Unit \\
\hline \multirow[t]{2}{*}{ Radiography } & Entrance surface dose, per view & ESD & mGy \\
\hline & Dose-area product, per examination & DAP & $\mathrm{Gy} \mathrm{cm}^{2}$ \\
\hline Mammography & Air Kerma at the breast surface, per view & ESAK & mGy \\
\hline Fluoroscopy & Dose-area product, per examination & DAP & $\mathrm{Gy} \mathrm{cm}^{2}$ \\
\hline Angiography and & Dose-area product, per examination & DAP & $\mathrm{Gy} \mathrm{cm} \mathrm{cm}^{2}$ \\
\hline \multirow[t]{2}{*}{ interventional radiology } & Number of images, per examination & - & - \\
\hline & Fluoroscopy time, per examination & - & $\min$ \\
\hline \multirow[t]{2}{*}{ Computed tomography } & Weighted CT dose index, per slice or rotation & $\mathrm{CTDI}_{\mathrm{w}}$ & mGy \\
\hline & Dose-length product, per examination & DLP & $\mathrm{mGy} \mathrm{cm}$ \\
\hline \multirow[t]{2}{*}{ Dental radiology } & $\begin{array}{l}\text { Entrance surface dose, per view for intra-oral } \\
\text { exminations (apical, bitewing) }\end{array}$ & ESD & mGy \\
\hline & Dose-width product for OPG & DWP & $\mathrm{mGy} \mathrm{mm}$ \\
\hline
\end{tabular}


in $\mathrm{mm}$ of aluminium, FSD is the focus-to-skin distance, and BSF is the back scattering factor. $K$ ( $\mathrm{mGy} \mathrm{m}^{2}$ per $\left.\mathrm{mAs}\right)$ is an empirically determined constant specifying the radiological unit; a typical value of $0.1 \mathrm{mGy} \mathrm{m}^{2}$ per mAs is adopted.

In the case of a fluoroscopy examination, the ESD takes the same form as above, $Q$ being replaced by the current $I$ (in $\mathrm{mA}$ ) times the exposure time $t$ (in $\mathrm{s}$ ). For this category of examinations, the operational dose quantity commonly used is the dose-area product (DAP usually expressed in $\mathrm{Gy} \mathrm{cm}^{2}$ ), which can be computed by multiplying the ESD by the field size at the entrance of the patient.

In the case of a CT examination, the operational dose quantity used for a slice is the weighted computed tomography dose index $\left(\mathrm{CTDI}_{\mathrm{w}}\right)$ measured in $\mathrm{mGy}$ representing the average dose that would be absorbed by the central slice within a $100 \mathrm{~mm}$ range of contiguous scanning, and defined as follows:

$$
\mathrm{CTDI}_{\mathrm{w}}=1 / 3 \mathrm{CTDI}_{\mathrm{c}}+2 / 3 \mathrm{CTDI}_{\mathrm{p}},
$$

where $\mathrm{CTDI}_{\mathrm{c}}$ (mGy) is measured at the centre of a homogeneous cylinder of polymethyl-methacrylate (PMMA), with diameters of $16 \mathrm{~cm}$ (head) or $32 \mathrm{~cm}$ (body), and $\mathrm{CTDI}_{\mathrm{p}}$ (mGy) is measured $10 \mathrm{~mm}$ below the surface of the phantom, and represents an average of measurements at four different locations around the periphery of that phantom.

The operational dose quantity used for a full CT examination is the dose-length product (DLP) measured in $\mathrm{mGy} \mathrm{cm}$ and defined as follows:

$$
\mathrm{DLP}=\mathrm{CTDI}_{\mathrm{w}} \cdot t \cdot n,
$$

Where $t(\mathrm{~cm})$ relates to the slice thickness and $n$ relates to the number of slices.

From the average values of the operational dose quantities, the DRLs are established by multiplying the average values by a factor 1.5 .

\section{RESULTS AND DISCUSSION}

The technical parameters related to the full set of 257 types of examinations and used to establish the corresponding DRLs are given in the detailed report of the 1998 nationwide survey available on-line ${ }^{(34)}$. In cases where the 1998 data are not used anymore, the technical parameters where modified accordingly.

As an example of radiographic examinations, Table 2 gives the average technical parameters for chest radiography (lateral view). The average ESD computed from these parameters equals $0.26 \mathrm{mSv}$. This leads, using the 1.5 multiplying factor, to a DRL of $0.4 \mathrm{mSv}$. This value is lower than the DRL of 1.5 proposed at the European level ${ }^{(35)}$. The technical parameters considered in the calculation are still in use. A survey that addressed four care-providing centres revealed that two of them use the same technical parameters, the third one uses a tube voltage of $150 \mathrm{kV}$, and the fourth one uses a tube voltage of $150 \mathrm{kV}$ and a current-time product of 14 mAs. This leads to a DRL of 0.56 and $1.6 \mathrm{mSv}$ respectively.

Concerning the examinations involving fluoroscopy, two examples are presented for illustration: barium meal (Table 3), Endoscopic retrograde cholangio pancreatography (ERCP) (Table 4) and cerebral angiography (Table 5).

Concerning barium mean, the technical parameters lead to a DRL in terms of total DAP of $110 \mathrm{~Gy} \mathrm{~cm}^{2}$, which is four-times higher than the $25 \mathrm{~Gy} \mathrm{~cm}^{2}$ value proposed at the European level ${ }^{(35)}$ and reported in the literature ${ }^{(36)}$, and about one order of magnitude higher than the figure published recently by NRPB $\left(13 \mathrm{~Gy} \mathrm{~cm}^{2}\right)^{(26)}$. A minimalist definition of this examination, i.e. 1 radiography

Table 2. Typical technical parameters for chest radiography (lateral view).

\begin{tabular}{lc} 
Tube voltage $(\mathrm{kVp})$ & 125 \\
Current-time product $(\mathrm{mAs})$ & 5 \\
Filtration $(\mathrm{mm} \mathrm{Al})$ & 3 \\
Focus-to-film distance $(\mathrm{cm})$ & 200 \\
Field size at the skin entrance plane $\left(\mathrm{cm}^{2}\right)$ & $30 \times 36$ \\
Sensitivity & 400 \\
Grid & Yes \\
\hline
\end{tabular}

\begin{tabular}{|c|c|c|c|}
\hline \multirow[t]{2}{*}{ Technical parameter } & \multicolumn{3}{|c|}{ Part of the body } \\
\hline & Oesophagus & Thorax & Abdomen \\
\hline Fluoroscopy time (s) & - & 120 & 300 \\
\hline Tube voltage $(\mathrm{kVp})$ & 65 & 70 & 80 \\
\hline Tube current (mA) & 1 & 2.5 & 3 \\
\hline Focus-to-image intensifier distance $(\mathrm{cm})$ & - & 60 & 60 \\
\hline Field size $\left(\mathrm{cm}^{2}\right)$ & $10 \times 12$ & $16 \times 35$ & $30 \times 40$ \\
\hline Number of images & 6 & 6 & 6 \\
\hline
\end{tabular}

Table 3. Typical technical parameters for barium meal. 


\section{A. AROUA ET AL}

Table 4. Typical technical parameters for ERCP.

\begin{tabular}{lc}
\hline & Abdomen \\
Part of the body exposed & 1200 \\
Fluoroscopy time $(\mathrm{s})$ & 80 \\
Tube voltage $(\mathrm{kVp})$ & 3 \\
Tube current $(\mathrm{mA})$ & 60 \\
Focus-to-image intensifier distance $(\mathrm{cm})$ & $30 \times 40$ \\
Field size $\left(\mathrm{cm}^{2}\right)$ & 8 \\
Number of images & 8 \\
\hline
\end{tabular}

Table 5. Typical technical parameters for cerebral
angiography.

\begin{tabular}{lc} 
Cine mode & \\
Tube voltage $(\mathrm{kVp})$ & 80 \\
Tube current $(\mathrm{mA})$ & 300 \\
Focus-to-image intensifier distance $(\mathrm{cm})$ & 100 \\
Number of sequences & 10 \\
Total number of images & 250 \\
Exposure time per image $(\mathrm{ms})$ & 25 \\
Total effective exposure time $(\mathrm{s})$ & 6.25 \\
Fluoroscopy & 70 \\
Tube voltage $(\mathrm{kVp})$ & 1.5 \\
Tube current $(\mathrm{mA})$ & 300 \\
Fluoroscopy time $(\mathrm{s})$ & 9 \\
Diameter of the image intensifier (in.) & 80 \\
Field size $\left(\mathrm{cm}^{2}\right)$ & \\
\hline
\end{tabular}

per region and $1 \mathrm{~min}$ of fluoroscopy for the thoracic and abdominal regions, would lead to a total DAP for the examination of $20 \mathrm{~Gy} \mathrm{~cm}^{2}\left(9.8 \mathrm{~Gy} \mathrm{~cm}^{2}\right.$ radiography and $10.5 \mathrm{~Gy} \mathrm{~cm}^{2}$ fluoroscopy), which is close to the figure reported in the literature $\left(20 \mathrm{~Gy} \mathrm{~cm}{ }^{2}\right)$. This seems too low to be representative of typical barium meal examination as it is practiced in Switzerland and which extends to the whole stomach. In other places, such as UK, barium meal is limited to the oesophagus region and involves five images only ${ }^{(26)}$, corresponding to the six images taken in Switzerland in the oesophagus part of barium meal. This explains the big differences found between the figure found here and the data of the literature. Another suspected source of discrepancy is a bad beam collimation when viewing the oesophagus region.

For ERCP, the result obtained here $\left(220 \mathrm{~Gy} \mathrm{~cm}^{2}\right)$ is extremely high compared to that found in the literature. This is due to the technical parameters used. In this work 8 images and $1200 \mathrm{~s}$ of fluoroscopy are considered, whereas in the UK for instance, the average figures are 4 images and $271 \mathrm{~s}$ of fluoroscopy ${ }^{(26)}$. An investigation with a few Swiss radiologists revealed that in Switzerland, ERCP is mainly a therapeutic (interventional) procedure and that diagnostic ERCP is no more performed by X rays but using other non-X-ray radiological modalities such as Magnetic resonance imaging (MRI). The
Table 6. Typical technical parameters for CT of the abdomen.

\begin{tabular}{lcc}
\hline Technical parameter & Series 1 & Series 2 \\
\hline Number of scans & 1 & 1 \\
Plane of first slice & $\begin{array}{l}\text { Hepatic } \\
\text { dome }\end{array}$ & Iliac crest \\
Plane of last slice & Iliac crest & Pubic symphysis \\
Mode & Helical & Axial \\
Slice thickness (mm) & 8 & 10 \\
Slice spacing (mm) & 1 & 0 \\
Pitch (overlap) & 1.12 & 1 \\
Length of scanned & 200 & 200 \\
volume (mm) & \multicolumn{2}{c}{} \\
Tube voltage (kVp) & 120 & 120 \\
Tube current (mA) & 220 & 260 \\
Rotation time (s) & 0.75 & 1 \\
CTDI $_{\mathrm{w}}$ (mG) & 11.4 & 17.8 \\
\hline
\end{tabular}

Table 7. Comparison of DRLs for a few radiographic views.

\begin{tabular}{lcc}
\hline Examination & $\begin{array}{c}\text { ESD (mGy) } \\
\text { this work }\end{array}$ & $\begin{array}{c}\text { ESD (mGy) } \\
\text { literature }\end{array}$ \\
\hline Skull PA/AP & 5.4 & 5 \\
Skull lateral & 3.5 & 3 \\
Cervical spine AP & 3.1 & 1.2 \\
Chest PA & 0.2 & $0.1-0.4$ \\
Chest lateral & 0.4 & 1.5 \\
Thoracic spine AP & 7 & 7 \\
Thoracic spine lateral & 21 & 20 \\
Abdomen AP & 7 & $4.3-10$ \\
Lumbar spine AP/PA & 8.7 & $3.9-10$ \\
Lumbar spine lateral & 26 & 30 \\
Pelvis AP & 7.8 & 10 \\
Hip AP & 4.7 & 10 \\
\hline
\end{tabular}

differences in DAP obviously come from differences in the definition of the examination.

As regards cerebral angiography the technical parameters lead to DRL of $50 \mathrm{~Gy} \mathrm{~cm}^{2}$. Padovani et al. ${ }^{(37)}$ reported recently a DRL value of $102 \mathrm{~Gy} \mathrm{~cm}^{2}$ established from a longer fluoroscopy time: $12 \mathrm{~min}$ instead of $5 \mathrm{~min}$ in this work.

As regards $\mathrm{CT}$ examinations, the protocol used for abdomen CT is presented in Table 6 . The technical parameters considered for this type of examination lead to a DRL in terms of CTDI ${ }_{w}$ per slice of $20 \mathrm{mGy}$ and in terms of DLP per examination of $710 \mathrm{mGy}$ $\mathrm{cm}$. These figures are slightly lower than the DRLs proposed at the European level ${ }^{(38)}$ : a CTDI ${ }_{w}$ per slice of $35 \mathrm{mGy}$ and DLP of $870 \mathrm{mGy} \mathrm{cm}$.

Tables 7-9 present the DRLs established for a number of examinations covering radiography, fluoroscopy and CT modalities and compared to the data reported in the literature. In the absence of detailed information concerning the technical 
DRLS FOR TEMPORARY USE IN SWITZERLAND

Table 8. Comparison of DRLs for a few examinations involving fluoroscopy.

\begin{tabular}{lccc}
\hline Examination & $\begin{array}{c}\text { DAP }\left(\mathrm{Gy} \mathrm{cm}^{2}\right) \\
\text { this work }\end{array}$ & $\begin{array}{c}\text { DAP }\left(\mathrm{Gy} \mathrm{cm}^{2}\right) \\
\text { literature }\end{array}$ & Reference \\
\hline IVU & 35 & 40 & $(24)$ \\
Barium meal & 110 & 25 & $(24)$ \\
Barium enema & 75 & 60 & $(24)$ \\
ERCP & 220 & 19.4 & $(39)$ \\
Hysterosalpingography & 10 & 4.1 & $(39)$ \\
Cerebral angiography & 50 & 102 & $(37)$ \\
Coronary angiography & 55 & $36-67$ & $(27,37,40)$ \\
Abdominal angiography & 90 & $139-265$ & $(41)$ \\
Renal angiography & 160 & 36 & $(47)$ \\
Lower limb angiography & 90 & 59 & $(40)$ \\
Cardiac catheterism & 122 & 64 & $(40)$ \\
Stent insertion & 84 & $90-130$ & $(27,37,42)$ \\
PTCA & 67 & 145 & $(40)$ \\
Valvuloplasty & 149 & $14-108$ & $(40)$ \\
Angioplasty & $14-155$ & $103-184$ & $(37,41)$ \\
Biliary drainage & 215 & 122 & $(40)$ \\
Pacemaker insertion & 38 & 123 & $(37)$ \\
Abdominal embolisation & 478 & &
\end{tabular}

Table 9. Comparison of DRLs for a few CT Examinations.

\begin{tabular}{|c|c|c|c|c|}
\hline \multirow[t]{2}{*}{ Examination } & \multicolumn{2}{|c|}{$\mathrm{CTDI}_{\mathrm{w}}$ per slice or rotation (mGy) } & \multicolumn{2}{|c|}{ DLP per examination ( $\mathrm{mGy} \mathrm{cm}$ ) } \\
\hline & This work & Literature $^{(24)}$ & This work & Literature $^{(24)}$ \\
\hline Head & 60 & 60 & 800 & 1050 \\
\hline Sinus & 30 & 35 & 510 & 360 \\
\hline Face & 30 & 35 & 510 & 360 \\
\hline Chest & 15 & 30 & 480 & 650 \\
\hline Abdomen & 20 & 35 & 710 & 780 \\
\hline Liver & 30 & 35 & 1170 & 900 \\
\hline Pelvis & 30 & 35 & 540 & 570 \\
\hline
\end{tabular}

parameters related to the DRLs reported in the literature that would allow a fine comparison of the results, one can assert that in general the two sets of data compare reasonably well, considering the level of variability in the techniques used, particularly in the case of complex examinations.

\section{CONCLUSIONS}

The results of the 1998 survey on the exposure of the public by diagnostic and interventional radiology in Switzerland were used to establish diagnostic reference levels for various types of radiological examinations. The average effective doses, calculated by dosimetric modelling using average technical parameters, were multiplied by a factor 1.5 to give results comparable to the DRL data determined by the 3rd-quartile method.

The comparison of the results obtained by this method with those reported in the literature show a satisfactory agreement for most cases, particularly simple examinations. For complex examinations, the discrepancies registered in some cases reflect the various sources of dose variability (how the examination is defined, differences in the techniques and protocols, the complexity of the case and the experience of the radiologist).

The DRLs presented are temporary, until the data collected through dosimetric surveys are available, and a national dosimetric database is set up which will allow the establishment of DRLs based on empirical data, as recommended at the international level.

\section{REFERENCES}

1. Sinnaeve, J. Quality control and radiation protection of the patient in diagnostic radiology and nuclear medicine. In: Proceedings of a Workshop held in Grado, Italy, 29 September-1 October 1993. Radiat. Prot. Dosim. 57(1-4), 1-2 (1995). 


\section{A. AROUA ET AL}

2. Lahure, J., Kaul, A. and Sinnaeve, J. Reference doses and quality in medical imaging. In: Proceedings of a Workshop held in Luxembourg, 23-25 October 1997, Radiat. Prot. Dosim. 80(1-3), 1-5 (1998).

3. Mattsson, S., Alpsten, M. and Herrmann, C. Medical $X$ ray imaging: potential impact of the new EC directive. In: Proceedings of a Workshop held in Malmö, Sweden, 13-15 June 1999. Radiat. Prot. Dosim. 90(1-2), 13-14 (2000).

4. Malone, J. F., Boyle, G., Marsh, D. M. and Tuenen, D. Dose and image quality in digital imaging and interventional radiology (DIMOND). In: Workshop held in Dublin, Ireland, 24-26 June 1999. Radiat. Prot. Dosim. 94(1-2), 9 (2001).

5. Duftschmid, K. E., Kusama, T. and Kase, K. R. Harmonisation of Radiation, Human Life and the Ecosystem. In: Proceedings of the 10th International Congress of the International Radiation Protection Association (IRPA-10), Hiroshima; 14-19 May (2000).

6. In: Proceedings of the International Conference on the Radiological Protection in Diagnostic and Interventional Radiology, Malaga 26-30 March 2001. IAEA C\&S Papers Series 7/P, Vienna (2001).

7. Gfirtner, H. Establishment of reference levels in diagnostic radiology. ERPET Course for Medical Physics. European Radiation Protection, Education and Training, Doc. RTD/0034/20 (2000).

8. Coursey, B. M., Taylor, D. M., Bradley, D. A., Ng, K. H., and Warren-Forward, H.M. Subject dose in radiological imaging. Appl. Radiat. Isotopes, 50(1), 1-275 (whole issue) (1999).

9. Wall, B. F. and Shrimpton, P. C. The historical development of reference doses in diagnostic radiology. Radiat. Prot. Dosim. 80(1-3), 15-20 (1998).

10. Stieve, F.-E. Historical development of reference levels in diagnostic radiology. ERPET Course for Medical Physics, Establishment of Reference Levels in Diagnostic Radiology, European Radiation Protection, Education and Training, Doc. RTD/0034/20 (2000)

11. Drexler, G. Diagnostic reference levels in the 1990 and 1996 Recommendations of the ICRP. Radiat. Prot. Dosim. 80(1-3), 7-10 (1998).

12. Oestmann, J.-W. The role and impact of reference doses in diagnostic radiology: problems and perspectives. Radiat. Prot. Dosim. 80(1-3), 21-22 (1998).

13. Broerse, J. J. and Geleijns, J. The relevance of different quantities for risk estimation in diagnostic radiology. Radiat. Prot. Dosim. 80(1-3), 33-37 (1998).

14. Veit, R., Bauer, B., Bernhardt, H. J. and Lechel, U. Proposed procedure for the establishment of diagnostic reference levels in germany. Radiat. Prot. Dosim. 80(1-3), 117-120 (1998).

15. Kalender, W. A. Computed tomography: influence of exposure parameters and the establishment of reference dose values. Radiat. Prot. Dosim. 80(1-3), 163-166 (1998).

16. Vañó, E. and González, L. Patient dosimetry and reference doses: practical considerations. Radiat. Prot. Dosim. 90(1-2), 85-88 (2000).

17. Connolly, P. A. and Moores, B. M. Appropriate methodology for reference levels: in diagnostic radiography. ERPET Course for Medical Physics, Establishment of Reference Levels in Diagnostic Radiology, European
Radiation Protection, Education and Training, Doc. RTD/0034/20 (2000).

18. Faulkner, K. Appropriate methodology for reference levels: in examinations using fluoroscopy. ERPET Course for Medical Physics, Establishment of Reference Levels in Diagnostic Radiology, European Radiation Protection, Education and Training, Doc. RTD/0034/20 (2000).

19. Vañó, E. Appropriate methodology for reference levels: in interventional radiology. ERPET Course for Medical Physics, Establishment of Reference Levels in Diagnostic Radiology, European Radiation Protection, Education and Training, Doc. RTD/0034/20 (2000).

20. Jessen, K. A. Appropriate methodology for reference levels: in CT. ERPET Course for Medical Physics, Establishment of Reference Levels in Diagnostic Radiology, European Radiation Protection, Education and Training, Doc. RTD/0034/20 (2000).

21. Säbel, M., Fitzgerald, M., Leitz, W. and Zoetelief, J. Appropriate methodology for reference levels: in mammography. ERPET Course for Medical Physics, Establishment of Reference Levels in Diagnostic Radiology, European Radiation Protection, Education and Training, Doc. RTD/0034/20 (2000).

22. Schneider, K. Appropriate Methodology for Reference Levels: In Paediatric Radiology. ERPET Course for Medical Physics, Establishment of Reference Levels in Diagnostic Radiology, European Radiation Protection, Education and Training, Doc. RTD/0034/20 (2000).

23. Zoetelief, J. Need for Harmonisation in the Establishment and Use of Reference Dose Levels in Radiology. In: Proceedings of the International Conference on the Radiological Protection in Diagnostic and Interventional Radiology, Malaga 26-30 March 2001. (Vienna: IAEA C\&S Papers Series 7/P), pp. 429-433, (2001).

24. International Commission on Radiological Protection. Diagnostic reference levels in medical imaging. ICRP Committee 3, Draft, 5 February (2001).

25. National Radiological Protection Board. National Protocol for Patient Dose Measurements in Diagnostic Radiology. Dosimetry Working Party of the Institute of Physical Sciences in Medicine, (Didcot: NRPB) (1992).

26. Hart, D., Hillier, M. C. and Wall, B. F. Doses to patients from medical $X$-ray examinations in the $U K-$ 2000 review. Report NRPB-W14, (Didcot: NRPB) (2002).

27. Neofotistou, V. Review of patient dosimetry in cardiology. Radiat. Prot. Dosim. 94(1), 177-182 (2001).

28. Mini, R. L. Dosimetric methods for the establishment of reference doses in conventional and computed radiography. Radiat. Prot. Dosim. 80(1-3), 221-224 (1998).

29. Mini, R. L., Schmid, B., Schneeberger, P. and Vock, P. Dose-area product measurements during angiographic $X$-ray procedures. Radiat. Prot. Dosim. 80(1-3), 145-148 (1998).

30. Verdun, F. R., Capasso, P., Valley J.-F. and Schnyder, P. Dose evaluation in fluoroscopy. Radiat. Prot. Dosim. 80(1-3), 139-141 (1998).

31. Verdun, F. R., Bernasconi, M., Schnyder, P., Valley, J. F. and Gudinchet, F. Introduction of guidance dose 


\section{DRLS FOR TEMPORARY USE IN SWITZERLAND}

levels in paediatric $C T$. In: Proceedings of the International Conference on the Radiological Protection in Diagnostic and Interventional Radiology, Malaga, 26-30 March 2001. (Vienna: IAEA C\&S Papers Series 7/P), pp 458-461, (2001).

32. Aroua, A., Buchillier-Decka, I., Vader, J. P. and Valley, J. F. Nationwide survey on radiation doses in diagnostic and interventional radiology in Switzerland in 1998. Health Phys. 83(1), 46-55 (2002).

33. Aroua A., Decka I., Burnand B., Vader J. P. and Valley J. F. Dosimetric aspects of a national survey of diagnostic and interventional radiology in Switzerland. Med. Phys. 29(10), 2247-2259 (2002).

34. Aroua, A., Besançon, A., Buchillier-Decka, I., Valley, J.-F. and Verdun, F. R. Niveaux de référence diagnostiques (NRD) pour la radiologie diagnostique et interventionnelle en Suisse. Institut de Radiophysique Appliquée, Lausanne (2002), Available at www.hospvd.ch/public/inst/ira.

35. European Commission. Guidance on diagnostic reference levels (DRLs) for medical exposures. Radiation Protection 109 (1999).

36. Corbett, R. H. The influence of radiologist's technique on patient dose in barium studies. In: Proceedings of the 10th International Congress of the International
Radiation Protection Association (IRPA-10), Hiroshima, 14-19 May (2000).

37. Padovani, R. and Maffessanti, M. Impact of EC directive 97/43 euratom in interventional radiology. Radiat. Prot. Dosim. 90(1-2), 39-45 (2000).

38. European Commission. Guidance on quality criteria for computed tomography. EUR 16262 (1999).

39. Broadhead, D. A., Chapple, C.-L. and Faulkner, K. Local reference doses during fluoroscopic procedures. Radiat. Prot. Dosim. 80(1-3), 143-144 (1998).

40. Broadhead, D. A., Chapple, C.-L., Faulkner, K., Davies, M. L. and McCallum, H. Local reference doses during cardiology procedures. Radiat. Prot. Dosim. 80(1-3), 149-150 (1998).

41. Ruiz Cruces, R., Garcia-Granados, J., Diaz Romero, F. J. and Hernández Armas, J. Estimation of effective dose in some digital angiographic and interventional procedures. Br. J. Radiol. 71, 42-47 (1998).

42. Widmark, A., Forsmark, H., Einarsson, G., Gundtoft, P., Hjardemaal, O., Leitz, W. and Pukkila, O. Guidance levels in the nordic countries: a preliminary report for selected interventional procedures. Radiat. Prot. Dosim. 94(1), 133-135 (2001). 